\title{
Expanding the role of proteasome homeostasis in Parkinson's disease: beyond protein breakdown
}

\author{
Mingxia Bi ${ }^{1}$, Xixun Du', Qian Jiao ${ }^{1}, X_{i}$ Chen $^{1}$ and Hong Jiang ${ }^{1}$
}

\begin{abstract}
Proteasome is the principal hydrolytic machinery responsible for the great majority of protein degradation. The past three decades have testified prominent advances about proteasome involved in almost every aspect of biological processes. Nonetheless, inappropriate increase or decrease in proteasome function is regarded as a causative factor in several diseases. Proteasome abundance and proper assembly need to be precisely controlled. Indeed, various neurodegenerative diseases including Parkinson's disease (PD) share a common pathological feature, intracellular protein accumulation such as a-synuclein. Proteasome activation may effectively remove aggregates and prevent the neurodegeneration in PD, which provides a potential application for disease-modifying treatment. In this review, we build on the valuable discoveries related to different types of proteolysis by distinct forms of proteasome, and how its regulatory and catalytic particles promote protein elimination. Additionally, we summarize the emerging ideas on the proteasome homeostasis regulation by targeting transcriptional, translational, and post-translational levels. Given the imbalanced proteostasis in PD, the strategies for intensifying proteasomal degradation are advocated as a promising approach for PD clinical intervention.
\end{abstract}

\section{Facts}

- Proteasome homeostasis undergoes dynamic and reversible regulation at transcriptional, translational, and post-translational levels.

- The vicious cycle between proteasome dysfunction and protein accumulation plays a key role in PD pathogenesis.

- Regulation of proteasome homeostasis serves as a promising approach in PD therapies.

- The development of PROTAC opens up new potentials for PD treatment based on proteasome modulation.

Correspondence: Hong Jiang (hongjiang@qdu.edu.cn)

${ }^{1}$ Department of Physiology, Shandong Provincial Key Laboratory of Pathogenesis and Prevention of Neurological Disorders and State Key

Disciplines: Physiology, School of Basic Medicine, Medical College, Qingdao

University, Qingdao, China

Edited by M. Agostini

\section{Open questions}

- A number of possible hybrid proteasomes arise in cells. What are the distinct functions of singly or doubly capped proteasomes?

- What are the mechanisms by which different transcription factors regulate a separate set of proteasome subunits?

- Is there a unified mechanism of protein aggregates and neurodegeneration triggered by proteasome dysfunction in Parkinson's disease?

- How prevalent and what is the significance of proteasome activation-based approach for $\mathrm{PD}$ therapies?

\section{Introduction}

Proteasome is a ubiquitous and highly plastic multisubunits complex responsible for protein degradation in both cytosol and nucleus. Since its initial discovery in $1987^{1,2}$, proteasome has been under intensive investigation to explore the inherent logic of proteasome function, 
including the recognition of ubiquitylated substrates, deubiquitylation of potential targets, translocation of unfolded proteins into the catalytic chamber, and the peptidase features for selective proteolysis ${ }^{3-5}$. Although widely assumed to be a single entity, there also exists various types of proteasome in cells, such as $26 \mathrm{~S}$ proteasome (constitutive proteasome), immunoproteasome, thymoproteasome, and spermatoproteasome ${ }^{6,7}$. Under certain conditions, specific proteasome is induced to perform different biological functions, while the efforts to distinguish these proteasomes and analyze their respective roles have not been accomplished. Indeed, proteasome is not a stable and static complex serving as a "cellular trashcan" ${ }^{8}$. The expression of proteasome undergoes dynamic and reversible regulation not only at the transcriptional level but also at the translational and posttranslational level, such as phosphorylation, ubiquitylation, ADP-ribosylation, O-linked $\mathrm{N}$-acetylglucosamine (O-GlcNAc), acetylation, and S-glutathionylation. Even with this information, a comprehensive understanding of the regulatory mechanisms underlying proteasome homeostasis remains elusive.

Proteasome activity becomes gradually impaired during aging and various neurodegenerative disorders including Parkinson's disease (PD). The compromise of proteasomal degradation leads to aberrant protein aggregates, such as $\alpha$-synuclein, which in turn binds to proteasome correlated with a reduction in protease activity ${ }^{9,10}$. These observations indicate a vicious cycle between proteasome dysfunction and protein accumulation in PD pathogenesis. It is thus inferred that proteasome is expected to be an attractive target for PD intervention ${ }^{11,12}$. Enhancing proteasome function by either increasing subunits assembly or activating $20 \mathrm{~S}$ gate opening might be useful for the clearance of protein aggregates. Consistent with this evidence, several small-molecule compounds have been postulated to be an effective approach in PD treatment. The development of proteolysis-targeting chimera (PROTAC) represents a burgeoning field and a new therapeutic regimen owing to the capacity of inducing a specific protein degradation, as well as its small-molecule nature and targeting "undruggable" proteins ${ }^{13,14}$. Additionally, PROTAC provides the opportunity to rapidly degrade proteins of interest, which effectively prevents the compensatory recovery due to the deletion of target proteins ${ }^{13,15}$. Even so, PROTAC is still an emerging technology, and its future development is also fraught with challenges.

This article reviews recent advances in the multiple regulation of proteasome homeostasis that ensures efficient protein degradation. Major new insights into proteasome activation have also emerged, which not only seems important in regulating protein turnover, but also provides potential application in drug discovery to combat the proteotoxicity in PD.

\section{Components of proteasome}

Proteasome, as a multisubunits complex, accounts for the vast majority (at least $80 \%$ ) of protein degradation. The best known $26 \mathrm{~S}$ proteasome, also named standard or constitutive proteasome, is composed of a $20 \mathrm{~S}$ core particle (CP or $20 \mathrm{~S}$ complex) attached to one or both ends by a $19 \mathrm{~S}$ regulatory particle (RP, $19 \mathrm{~S}$ complex or PA700). The $19 \mathrm{~S}$ complex, as a proteasome activator, is incorporated into two parts: the lid and the base. The lid consists of nine subunits, that is, Rpn3, Rpn5, Rpn6, Rpn7, Rpn8, Rpn9, Rpn11, Rpn12, and Rpn15. The base contains six ATPase subunits (Rpt1-6), as well as Rpn1, Rpn2, Rpn10, and Rpn13 (Table 1). The alternative proteasome activators, including $11 \mathrm{~S}$ regulator complex PA28 and PA200 (PSME4), substitute 19 S RP to assemble various forms of proteasome. Three members of PA28 have been identified, namely PA28 $\alpha$ (PSME1), PA28 $\beta$ (PSME2), and PA28 $\gamma$ (PSME3). In contrast to PA28 $\alpha$ and PA28 $\beta$ which form PA28 $\alpha \beta$ hetero-heptamer distributed throughout the cytoplasm, PA28y forms a homo-heptamer predominantly located in the nucleus. Many studies have implicated PA28 $\alpha \beta$ in the production of major histocompatibility complex class I (MHC I) antigen peptides, and PA28y has been known to conduct proteasomal degradation of numerous intact proteins ${ }^{16,17}$.

The $20 \mathrm{~S}$ complex is a barrel-shaped hollow cylindrical complex with 28 subunits arranged into four heptameric rings, the two outer rings composed of $\alpha_{1-7}$ subunits and the two inner rings composed of $\beta_{1-7}$ subunits. Of note, the proteolytic activities are carried out by three $\beta$ subunits with peptidase sites, namely $\beta 1, \beta 2$, and $\beta 5$, which possesses the caspase-like, trypsin-like, and chymotrypsin-like activities, respectively. Upon certain stimulus, three catalytic subunits $\beta 1, \beta 2$, and $\beta 5$ subunits can be replaced by their homologues $\beta 1 \mathrm{i}, \beta 2 \mathrm{i}$, and $\beta 5 \mathrm{i}$ to form immunoproteasome ${ }^{18}$, $\beta 5$ t substitutes $\beta 5$ to form thymoproteasome ${ }^{19}$, and $\alpha 4 \mathrm{~s}$ subunits replace their constitutive counterparts $\alpha 4$ to form spermatoproteasome ${ }^{20}$. Currently, a number of possible hybrid proteasomes arise in cells because of the attachment of different proteasome activators to one or both sides of $20 \mathrm{~S} \mathrm{CP}$ (Fig. 1). Nonetheless, the specific function of singly or doubly capped proteasomes remains to be elucidated.

\section{Regulation of proteasome homeostasis}

\section{Transcriptional regulation of constitutive proteasome}

In 1999, Mannhaupt et al. discovered a unique activating sequence (5'-GGTGGCAAA-3') called proteasomeassociated control element (PACE), which is localized in the promoters of most proteasome subunits ${ }^{21}$. In yeast, Rpn4 acts as a common transcription factor binding to PACE of genes encoding proteasome subunits to maintain the normal abundance of proteasome $\mathrm{e}^{21-23}$. Notably, Rpn4 is extremely short-lived $\left(\mathrm{t}_{1 / 2} \sim 2 \mathrm{~min}\right)$ because the 
Table 1 Proteasome subunits names and function.

\begin{tabular}{|c|c|c|c|}
\hline Subcomplex & $\begin{array}{l}\text { Saccharomyces } \\
\text { cerevisiae }\end{array}$ & $\begin{array}{l}\text { Homo } \\
\text { sapiens }\end{array}$ & Function \\
\hline \multirow[t]{9}{*}{$19 \mathrm{~S} \mathrm{lid}$} & Rpn3 & PSMD3 & Structural \\
\hline & Rpn5 & PSMD12 & Structural \\
\hline & Rpn6 & PSMD11 & Structural \\
\hline & Rpn7 & PSMD6 & Structural \\
\hline & Rpn8 & PSMD7 & Structural \\
\hline & Rpn9 & PSMD13 & Structural \\
\hline & Rpn11 & PSMD14 & $\begin{array}{l}\text { Deubiquitinase, Ub } \\
\text { removal }\end{array}$ \\
\hline & Rpn12 & PSMD8 & Structural \\
\hline & Rpn15 & PSMD9 & Structural \\
\hline \multirow[t]{10}{*}{$19 S$ base } & Rpn1 & PSMD2 & $\begin{array}{l}\text { Ubp6 and Ub/Ubl } \\
\text { binding }\end{array}$ \\
\hline & Rpn2 & PSMD1 & Structural \\
\hline & Rpn10 & PSMD4 & Initial Ub/Ubl binding \\
\hline & Rpn13 & ADRM1 & \\
\hline & Rpt1 & PSMC2 & \multirow{6}{*}{$\begin{array}{l}\text { ATPase subunits, } \\
\text { substrates binding, } \\
\text { unfolding, and } \\
\text { translocation }\end{array}$} \\
\hline & Rpt2 & PSMC1 & \\
\hline & Rpt3 & PSMC4 & \\
\hline & Rpt4 & PSMC6 & \\
\hline & Rpt5 & PSMC3 & \\
\hline & Rpt6 & PSMC5 & \\
\hline \multirow[t]{7}{*}{$20 \mathrm{~S}$ a-subunits } & a1 & PSMA6 & \multirow[t]{7}{*}{$20 \mathrm{~S}$ gate opening } \\
\hline & a2 & PSMA2 & \\
\hline & a3 & PSMA4 & \\
\hline & $a 4$ & PSMA7 & \\
\hline & a5 & PSMA5 & \\
\hline & $a 6$ & PSMA1 & \\
\hline & a7 & PSMA3 & \\
\hline \multirow[t]{10}{*}{$20 S \beta$-subunits } & $\beta 1$ & PSMB6 & Caspase-like activity \\
\hline & $\beta 1 i$ & PSMB9 & $\begin{array}{l}\text { Chymotrypsin-like } \\
\text { activity }\end{array}$ \\
\hline & $\beta 2$ & PSMB7 & Trypsin-like activity \\
\hline & $\beta 2 i$ & PSMB10 & \multirow[t]{3}{*}{ Trypsin-like activity } \\
\hline & $\beta 3$ & PSMB3 & \\
\hline & $\beta 4$ & PSMB2 & \\
\hline & $\beta 5$ & PSMB5 & $\begin{array}{l}\text { Chymotrypsin-like } \\
\text { activity }\end{array}$ \\
\hline & $\beta 5 i$ & PSMB8 & \multirow[t]{3}{*}{$\begin{array}{l}\text { Chymotrypsin-like } \\
\text { activity }\end{array}$} \\
\hline & $\beta 6$ & PSMB1 & \\
\hline & $\beta 7$ & PSMB4 & \\
\hline \multirow[t]{2}{*}{ Associated DUBs } & Ubp6 & USP14 & \multirow{2}{*}{$\begin{array}{l}\text { Deubiquitinase, } 19 \mathrm{~S} \\
\text { activation }\end{array}$} \\
\hline & - & UCH37 & \\
\hline
\end{tabular}

$\mathrm{N}$-terminal region of Rpn4 contains a portable degradation signal for the continual proteasomal degradation. As a consequence, Rpn4 augments the synthesis of proteasome subunits in response to proteasome inhibition, which yields a negative feedback circuit. By contrast, Rpn4 deletion in yeast disrupts the expression of proteasome, therefore cells become more susceptible to various stimulus such as DNA damage and oxidative stress ${ }^{24}$. In yeast, transcription factor Rpn4 is indispensable for the compensatory increase of proteasome subunits to cope with stress conditions (Fig. 2).
In mammals, there also exists the transcriptional regulation of proteasome expression once proteasome function is impaired (Fig. 2). Despite the homologues of Rpn4 in mammalian cells have not been found, several transcription factors are implicated to fulfill the function of Rpn4. The expression of proteasome genes, including six $\mathrm{CP}$ subunits $(\alpha 2, \alpha 5, \alpha 7, \beta 3, \beta 4$, and $\beta 6)$ and five $R P$ subunits (Rpt1, Rpt5, Rpt6, Rpn10, and Rpn11), is tightly regulated by nuclear transcription factor $\mathrm{Y}$ (NF-Y). Knockdown of NF-Y remarkably downregulates proteasome genes expression and inhibits cellular proteasome function $^{25}$. Forkhead box O4 (FOXO4), an insulin/insulin-like growth factor-I (IGF-I) responsive transcription factor, amplifies proteasome activity by modulating Rpn6 expression in human embryonic stem cells ${ }^{26}$. Signal transducer and activator of transcription 3 (STAT3), which is activated through Janus kinase (JAK)/STAT pathway, transcriptionally regulates the expression of $\beta 5^{27}$. In addition, nuclear factor erythroid 2-related factor 2 (NRF2) is initially implicated to induce $\beta 5$ expression upon exposure to oxidative stress ${ }^{28}$. Later, antioxidant response elements (AREs) sequences have been found in the 5 -untranslated region of $20 \mathrm{~S}$ proteasome subunits genes $^{29}$. Intriguingly, a later study ascribes the induction of proteasome biogenesis to $\mathrm{NRF}^{30}$. Echoing these results, brain-specific $\mathrm{Nrf1}^{-1-}$ knockout mice exhibit the downregulation of proteasomal genes, accompanied by the accumulation of polyubiquitylated proteins and agedependent neurodegeneration ${ }^{31}$.

\section{Transcriptional regulation of immunoproteasome}

Unlike the constitutive proteasome, immunoproteasome subunits lack functional ARE binding sequences. The $L M P 2$ gene encoding $\beta 1 \mathrm{i}$ subunit contains a bidirectional promoter characterized by the lack of TATA box and the presence of several GC boxes, which are likely the transcriptional start sites. Multiple transcription factors including signal transducer and activator of transcription 1 (STAT1)/interferon regulatory factor 1 (IRF1) dimers, nuclear factor $\kappa \mathrm{B}$ (NF- $\mathrm{kB}$ ), SP-1, AP-1, cAMP responsive element binding protein (CREB), and Zif268 (also known as Egr1), are involved in the LMP2 ( $\beta 1 \mathrm{i})$ gene expres$\operatorname{sion}^{32}$. Furthermore, the promoter regions of LMP7 and $M E C L-1$ encoding $\beta 5 \mathrm{i}$ and $\beta 2 \mathrm{i}$, respectively, also contain $\mathrm{NF}-\mathrm{KB}$ consensus sequence, cAMP regulatory elements, along with SP-1 and IRF1 binding sites ${ }^{18,33,34}$. In this case, the transcriptional regulation of $\beta 1 \mathrm{i}, \beta 2 \mathrm{i}$, and $\beta 5 \mathrm{i}$ share the relatively similar mechanisms. Upon interferon- $\gamma$ (IFN- $\gamma$ ) stimulation, the activation of JAK1 and JAK2 causes the dimerization and phosphorylation of STAT1, which translocate into the nucleus and combine with IRF1 to promote its transcription. Then, IRF1 migrates back into the nucleus to stimulate the expression of immunoproteasome subunits. In addition, a potential alternative 


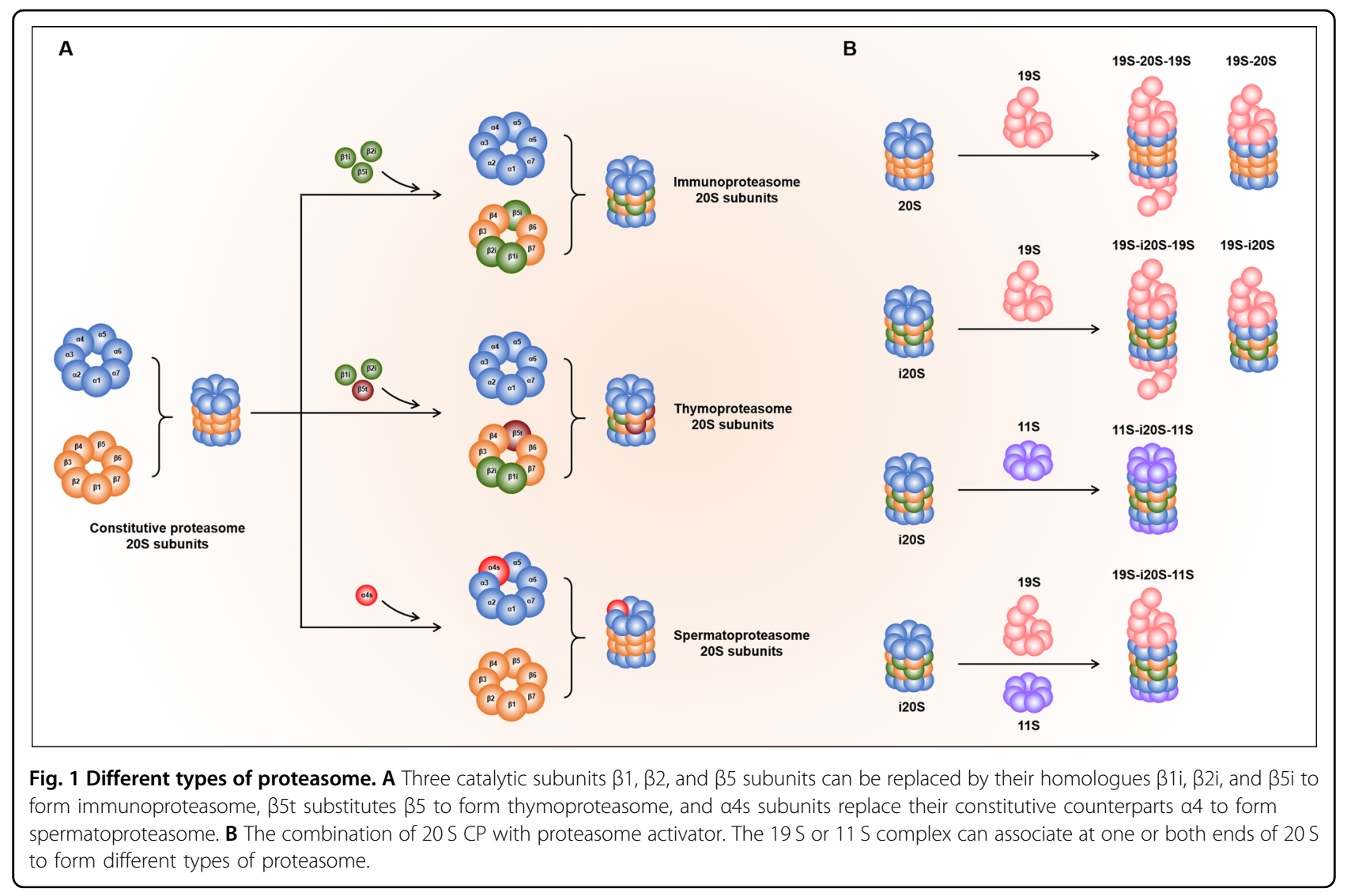

manner for immunoproteasome regulation is through NF- $\mathrm{kB}$ pathway. Upon oxidative injury, the phosphorylation of protein kinase $D(P K D)$ disassociates $I \kappa B \alpha$ from NF- $k B$. Upon the degradation of I $\mathrm{KB} \alpha$ by the proteasome, $\mathrm{NF}-\mathrm{kB}$ can translocate into the nucleus triggering the transcription of immunoproteasome subunits (Fig. 2).

\section{Translational regulation of proteasome}

The activation of yeast mitogen-activated protein kinase (MAPK) Mpk1 followed by target of rapamycin complex 1 (TORC1) inhibition facilitates a rapid rise in the expression of RP assembly chaperones (RACs) and proteasome subunits. This process of proteasome homeostasis regulation is evolutionarily conserved in mammals. ERK5 (also known as MAPK7), the mammalian orthologue of Mpk1, also mediates the upregulation of RACs and proteasome abundance upon mammalian target of rapamycin complex 1 (mTORC1) inhibition ${ }^{35}$. Considering that neither the mRNA levels nor the protein stability of proteasome subunits are altered in response to the inhibition of TORC1/mTORC1, the regulation of proteasome by Mpk1/ERK5 probably occurs at the translational level $^{36}$. It is clear that mTORC1 serves as a master regulator of proteasome abundance, whereas the relationship between mTORC1 and proteasome homeostasis seems to be controversial. Another study reveals that mTORC1 activation promotes the efficiency of proteasomemediated protein degradation by increasing cellular proteasome content ${ }^{37}$. In this regard, it will be necessary to resolve the discrepancy of how mTORC1 affects the proteasome homeostasis under the particular cellular conditions.

\section{Post-translational modifications of proteasome Phosphorylation}

In 1989, Haass and Kloetzel first reported the possibility that the phosphorylation of proteasome subunits had an impact on proteolytic activities during the Drosophila development ${ }^{38}$. In the following years, phosphorylation proteomics have shown a great deal of phosphorylation sites, which exist in almost every proteasome subunit ${ }^{8}$. Protein kinase A (PKA) was probably the first reported kinase involved in the phosphorylation of proteasome subunits $^{39}$. Subsequent studies have shown that PKA directly phosphorylate Rpt6 at Ser120 and Rpn6 at Ser14, leading to the increased proteasomal peptidase activities $^{40-42}$. PKA activation enhances the capacity of proteasome and promotes the elimination of protein aggregates $^{41}$. In rat spinal cord neurons, PKA-mediated increased proteolytic activities reduce the accumulation of ubiquitylated proteins and protect cells from inflammatory injury ${ }^{43} \cdot \mathrm{Ca}^{2+} /$ calmodulin-dependent protein kinase 
A Transcriptional regulation of proteasome in yeast

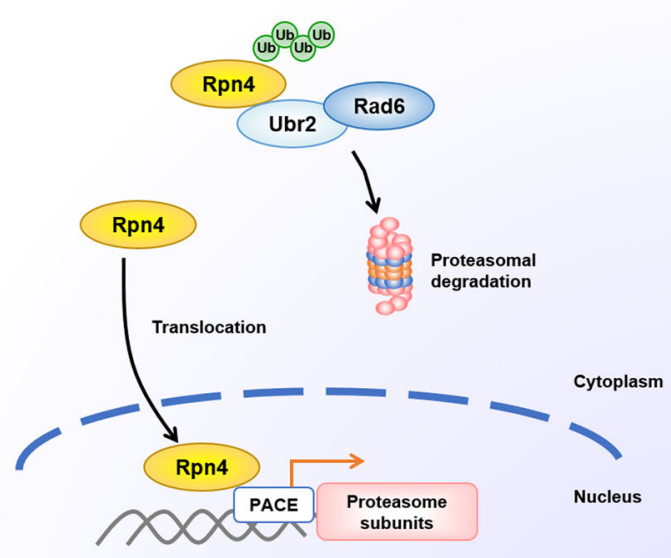

B

\section{Transcriptional regulation of constitutive proteasome in mammals}

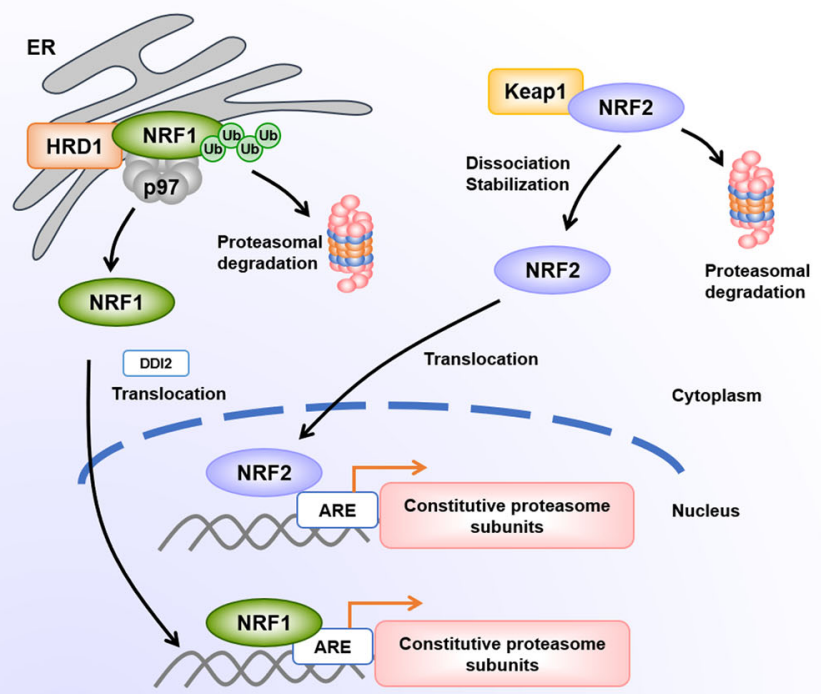

C

Transcriptional regulation of immunoproteasome in mammals

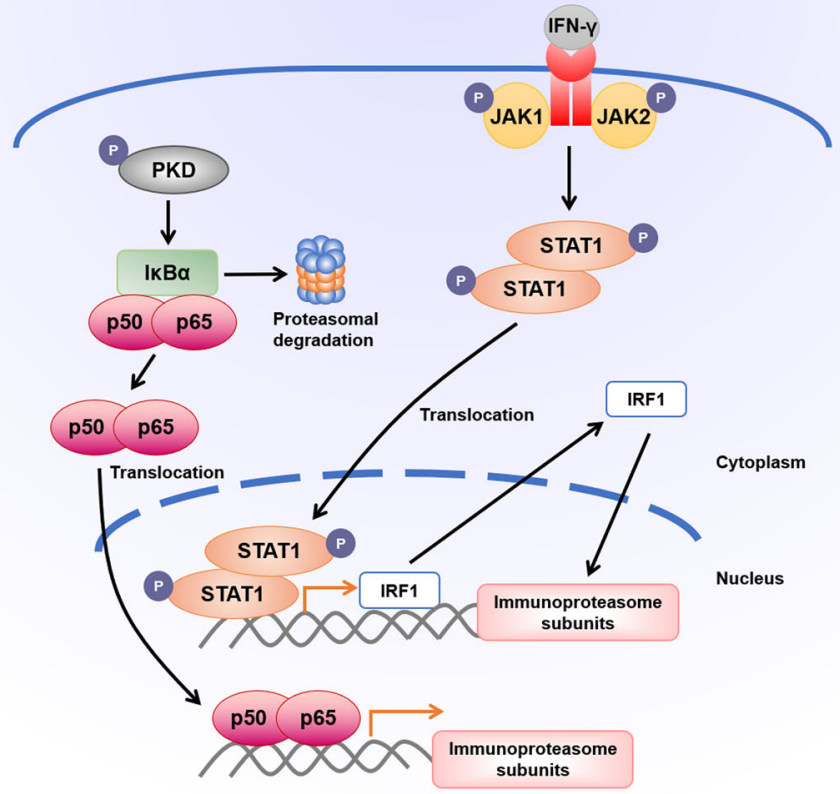

Fig. 2 Transcriptional regulation of proteasome expression. A Transcriptional regulation of proteasome in yeast. Rpn4 serves as a transcription factor with short half-life (t1/2 2 min) owing to the proteasomal degradation mediated by E2 ubiquitin conjugating enzyme Rad6 and E3 ubiquitin ligase Ubr2. Upon proteasome inhibition, Rpn4 is translocated into the nucleus, where it binds to PACE sequence in the promoters of proteasome subunit genes, resulting in the compensatory increase of proteasome expression. B Transcriptional regulation of constitutive proteasome in mammals. NRF1 resides in the ER, which is degraded via ERAD pathway requiring ER-resident ubiquitin ligase HRD1 and ATPase p97. When the proteasome is inhibited, NRF1 is cleaved by DDI2, and then translocated to the nucleus where it binds ARE and activates the transcription of proteasome genes. During oxidative stress, NRF2-Keap1 complex disassociates, and NRF2 translocates into the nucleus to transcriptionally regulate proteasome expression. C Transcriptional regulation of immunoproteasome in mammals. Under the stimulation of IFN- $\gamma$, activated JAK1 and JAK2 leads to the dimerization and phosphorylation of STAT1, which translocates into the nucleus and binds to IRF1. Following translation, IRF1 moves back into the nucleus to increase the transcription of immunoproteasome. Upon oxidative injury, phosphorylated protein kinase D (PKD) leads to the disassociation of IKBa from NF-KB. In turn, IKBa is degraded and NF-KB translocates into the nucleus to regulate the expression of immunoproteasome. 
Table 2 An overview of proteasome related post-translational modifications.

\begin{tabular}{|c|c|c|c|c|}
\hline Modifications & Targets & Enzymes & Functions & References \\
\hline \multirow[t]{8}{*}{ Phosphorylation } & Rpn1 (S361) & UBLCP1 & Proteasome activity $\uparrow$; $26 \mathrm{~S}$ proteasome assembly $\uparrow$ & 146 \\
\hline & Rpn2 (Y273) & p38 MAPK & Proteasome activity $\downarrow$; ubiquitylated protein degradation $\downarrow$ & 52 \\
\hline & Rpn6 (S14) & PKA & Proteasome activity $\uparrow$; ubiquitylated protein degradation $\uparrow$ & 41,42 \\
\hline & Rpt3 (T25) & DYRK2 & $\begin{array}{l}\text { Proteasome activity } \uparrow \text {; substrate translocation and degradation } \uparrow \text {; } \\
\text { cell proliferation and tumorigenesis }\end{array}$ & 50,51 \\
\hline & Rpt6 (S120) & PKA, CAMKIla & Proteasome activity $\uparrow$; synaptic plasticity, learning and memory & 40,41 \\
\hline & a4 (Y153) & $\mathrm{c}-\mathrm{Abl} / \mathrm{Arg}$ & Proteasome activity $\downarrow$ & 53 \\
\hline & $a 4(Y 106)$ & c-Abl/Arg & a4 degradation $\downarrow$; proteasome abundance $\uparrow$ & 54 \\
\hline & a7 $(\$ 243, S 250)$ & CK2 & Stabilizing RP-CP interaction; Ecm29 binding $\uparrow$ & 55,147 \\
\hline \multirow[t]{3}{*}{ Ubiquitylation } & Rpn10 & Ube3c, Rsp5, Ubp2 & Proteasome activity $\downarrow$; ubiquitylated conjugates affinity $\downarrow$ & 56,57 \\
\hline & Rpn13 & Ube3c & Ubiquitylated protein degradation $\downarrow$ & 58 \\
\hline & $a 2$ & ALAD & Substrates entry into $20 \mathrm{~S} C P \downarrow$ & 59 \\
\hline ADP-ribosylation & $\begin{array}{l}\text { Nuclear } \\
\text { proteasome }\end{array}$ & PARP & $\begin{array}{l}\text { Proteasome activity } \uparrow \text {; oxidatively histone degradation } \uparrow \text {; } \\
\text { cytokine-mediated neuroinflammation } \downarrow\end{array}$ & 60,61 \\
\hline O-GICNAC & Rpt2 & OGT, OGA & Proteasome activity $\downarrow$; ubiquitylated protein degradation $\downarrow$ & 65 \\
\hline Acetylation & $a 6, \beta 3, \beta 6, \beta 7$ & HDAC & Proteasome activity $\uparrow$ & 67 \\
\hline \multirow[t]{2}{*}{ S-glutathionylation } & Rpn1, Rpn2 & - & Proteasome activity $\downarrow$ & 148 \\
\hline & $a 5, a 6, a 7$ & $\begin{array}{l}\text { Glutaredoxin 2, } \\
\text { thioredoxins } 1 / 2\end{array}$ & $20 S$ gate opening $\uparrow$; oxidized and unfold protein degradation $\uparrow$ & 149,150 \\
\hline
\end{tabular}

II (CAMKII $\alpha)$ directly phosphorylates Rpt6 at Ser120 and stimulates proteasome activity ${ }^{44,45}$. Mutation of Rpt6 at Ser120 blocks proteasome-dependent regulation of synaptic plasticity in the hippocampus ${ }^{46,47}$ (Table 2). Pharmacological inhibition of CaMKII $\alpha$ abolishes the increase in proteolytic activity and the initiation of memory reconsolidation process ${ }^{48,49}$.

Dual-specificity tyrosine-regulated kinase 2 (DYRK2) phosphorylates a particular site of the proteasome, Rpt3 Thr25, leading to the enhanced substrate translocation and degradation ${ }^{50,51}$. Under osmotic stress conditions, p38 MAPK negatively regulates the proteasome activity by phosphorylating Rpn2 at Thr273, so as to induce the accumulation of polyubiquitinated proteins ${ }^{52}$. The nonreceptor tyrosine kinases c-Abl and Arg directly phosphorylate $\alpha 4$ at two tyrosine residues, Tyr106 and Tyr153. $\alpha 4$ Tyr153 phosphorylation inhibits proteasome activity, whereas Tyr106 phosphorylation suppresses the degradation of $\alpha 4$, therefore upregulating proteasome abundance $^{53,54}$. These findings suggests the dual roles of c$\mathrm{Abl} / \mathrm{Arg}$ in the proteasome homeostasis and activity. Moreover, casein kinase 2 (CK2) phosphorylates $\alpha 7$ at Ser243 and Ser250, which appears to stabilize the RP-CP interaction and promote the binding of proteasome quality control factor Ecm $29^{55}$ (Table 2).

\section{Ubiquitylation}

Rpn10 is the first proteasome subunit identified as a proteasome substrate, as well as a major target of ubiquitylation. It has been shown that Rpn10 undergoes the proteasomal degradation facilitated by E3 ubiquitin ligase Ube $3 c^{56}$. Indeed, Ube3c endows proteasomes with the capacity to extend the ubiquitin chains on targets, which can be disassembled by a proteasome-associated deubiquitinating enzymes (DUB), Ubp6. Moreover, Isasa et al. have reported that Rpn10 is ubiquitylated by Rsp5 and deubiquitylated by Ubp2. Under stress conditions, Rpn10 monoubiquitylation is reduced, which in turn rescues proteasome function by enhancing the proteolytic activity, providing a stress sensitive mechanism to control proteasome catalytic activity ${ }^{57}$. Additionally, when proteasome function is inhibited by bortezomib, Rpn13 becomes extensively and selectively polyubiquitylated by E3 ligase Ube3c, which strongly inhibits the proteasome's ability to degrade ubiquitin conjugates, but not hydrolyzing peptides and non-ubiquitylated proteins ${ }^{58}$ (Table 2). It has also been reported that delta-aminolevulinic acid dehydratase (ALAD) serves as an endogenous proteasome inhibitor, which is associated with the ubiquitylation of $\alpha 2$ subunit, thereby inhibiting the entry of substrates into $20 \mathrm{~S}$ catalytic chamber $^{59}$. Although the consequence of proteasome 
subunits ubiquitylation under different conditions remain a subject for further study, this modification appears to be valuable to evaluate the proteasome function.

\section{ADP-ribosylation}

ADP-ribosylation is the transfer of ADP-ribose moiety from $\mathrm{NAD}^{+}$to target proteins. The functional interaction of poly(ADP-ribose) with $20 \mathrm{~S}$ proteasome leads to a specific enhancement of the peptidase activity. ADPribosylation activates nuclear $20 \mathrm{~S}$ proteasome to efficiently degrade oxidatively modified histone proteins, which is proposed as an adaptive response to oxidative defense $^{60}$. Given the important role of ADP-ribosylation in DNA repair, the activation of proteasome is accompanied by the degradation of oxidized histones in the nucleus, which will otherwise make DNA repair impossible $^{61}$. Besides, proteasome modification via ADPribosylation participates in cytokine-mediated neuroinflammation. Poly(ADP-ribose) polymerase (PARP) enables activated microglia to resist oxidative injury through an upregulation of the nuclear proteasome activity, resulting in the enhanced protein turnover and degradation of oxidatively modified proteins ${ }^{62}$. Of note, PARP inhibition impairs activated, but not resting microglia owing to the reduced proteasomal degradation, which might be beneficial, particularly in PD in which activated microglia acts as a pathogenic factor. Considering that glial cells are involved in the maintenance of neuronal function, PARP might be taken into consideration with the intention of ensuring neuronal survival.

\section{O-Glycosylation}

O-GlcNAc is a regulatory post-translational modification in which O-GlcNAc transferase (OGT) adds GlcNAc monosaccharides to the hydroxyl groups on serine or threonine residues, while O-GlcNAcase (OGA) removes this modification ${ }^{63}$. Both OGT and OGA are abundant in the brain, with the highest expression in the hippocampal granular and pyramidal neurons and cerebellar Purkinje cells $^{64}$. Pharmacological inhibition of OGA activity by streptozotocin or OGA knockdown leads to a rapid accumulation of O-GlcNAc and inhibition of proteasome function, thereby exacerbating neuronal apoptosis. Exposure of $26 \mathrm{~S}$, but not $20 \mathrm{~S}$, proteasome to OGT impairs the proteolysis of transcription factor SP-1 and a hydrophobic peptide owing to the inhibition of $19 \mathrm{~S}$ ATPase activities. The degradation of ubiquitinylated proteins requires the opening of $20 \mathrm{~S} \mathrm{CP}$ probably by Rpt2, whose O-GlcNAc modification correlates inversely with the proteasome activity ${ }^{65}$ (Table 2 ). In addition, the biotin-cystamine tag strategy identifies six O-GlcNAc sites within the murine $20 \mathrm{~S} \mathrm{CP}$, that is, $\alpha 1$ (Ser5), $\alpha 4$ (Ser130), $\alpha 5$ (Ser198), $\alpha 6$ (Ser110), and $\beta 6$ (Ser57 and Ser208). O-GlcNAc sites of $\alpha 1$ and $\alpha 5$ are only found in immunoproteasomes, whereas $\beta 6$ Ser208 modification is detected only in brain proteasomes ${ }^{66}$. O-GlcNAc modification may serve as an important regulation system in the proteasome homeostasis, and investigating the biological impact of O-GlcNAc on proteasome seems to be a challenging but promising field of research.

\section{Acetylation}

Acetylation is a reversible post-translational modification of proteins, regulated by histone acetyltransferases (HATs) and histone deacetylases (HDACs), which add and remove acetyl groups from lysine residues, respectively. Despite the well-documented function of acetylation in modulating gene transcription and protein expression, its role in protein degradation has been recognized. Five lysine acetylation sites on murine $20 \mathrm{~S}$ subunits are identified following HDAC inhibition, that is, Lys30 and Lys115 of $\alpha 6$ subunit, Lys77 of $\beta 3$ subunit, Lys203 of $\beta 6$ subunit, and Lys 201 of $\beta 7$ subunit (Table 2). The enhanced acetylation of $20 \mathrm{~S}$ proteasome subunits leads to an elevation of proteolytic capacity ${ }^{67}$. In this regard, HDAC inhibitors might be a promising class of pharmacological agents for increasing the proteasomal proteolytic activity. Beyond that, six Lys residues (Lys6, Lys11, Lys27, Lys33, Lys48, and Lys63) on ubiquitin have been reported to be acetylated in proteomics datasets ${ }^{68}$. Acetylated ubiquitin does not affect the monoubiquitylation of substrates, but inhibits Lys11, Lys48, and Lys63linked polyubiquitin chains elongation ${ }^{69}$. Ubiquitin is subject to acetylation to modulate protein degradation, thus providing a new regulatory layer to ubiquitinproteasome biology. Further research needs to identify the HATs and HDACs responsible for proteasome acetylation and deacetylation, as well as functional roles of acetylated ubiquitin.

\section{Imbalance of proteostasis in PD \\ Proteasome dysfunction exacerbates protein aggregates in PD}

In PD, the selective loss of dopaminergic neurons in the substantia nigra pars compacta ( $\mathrm{SNpc}$ ) and subsequent loss of dopamine in the striatum leads to the classical motor features, such as resting tremor, bradykinesia, rigidity, and postural instability. Although the exact pathogenesis of PD has not been revealed yet, it is generally accepted that disruption of cellular proteostasis is linked to various neurodegenerative diseases including $\mathrm{PD}^{70}$. In eukaryotic cells, two major protein clearance pathways, proteasome and autophagy, are interrelated to maintain cellular proteolysis and ensure that cells have the adequate proteins they need ${ }^{71,72}$. The proteasome, in collaboration with a refined ubiquitin system, selectively degrades short-lived proteins as well as misfolded or damaged proteins in the cytoplasm, nucleus and 


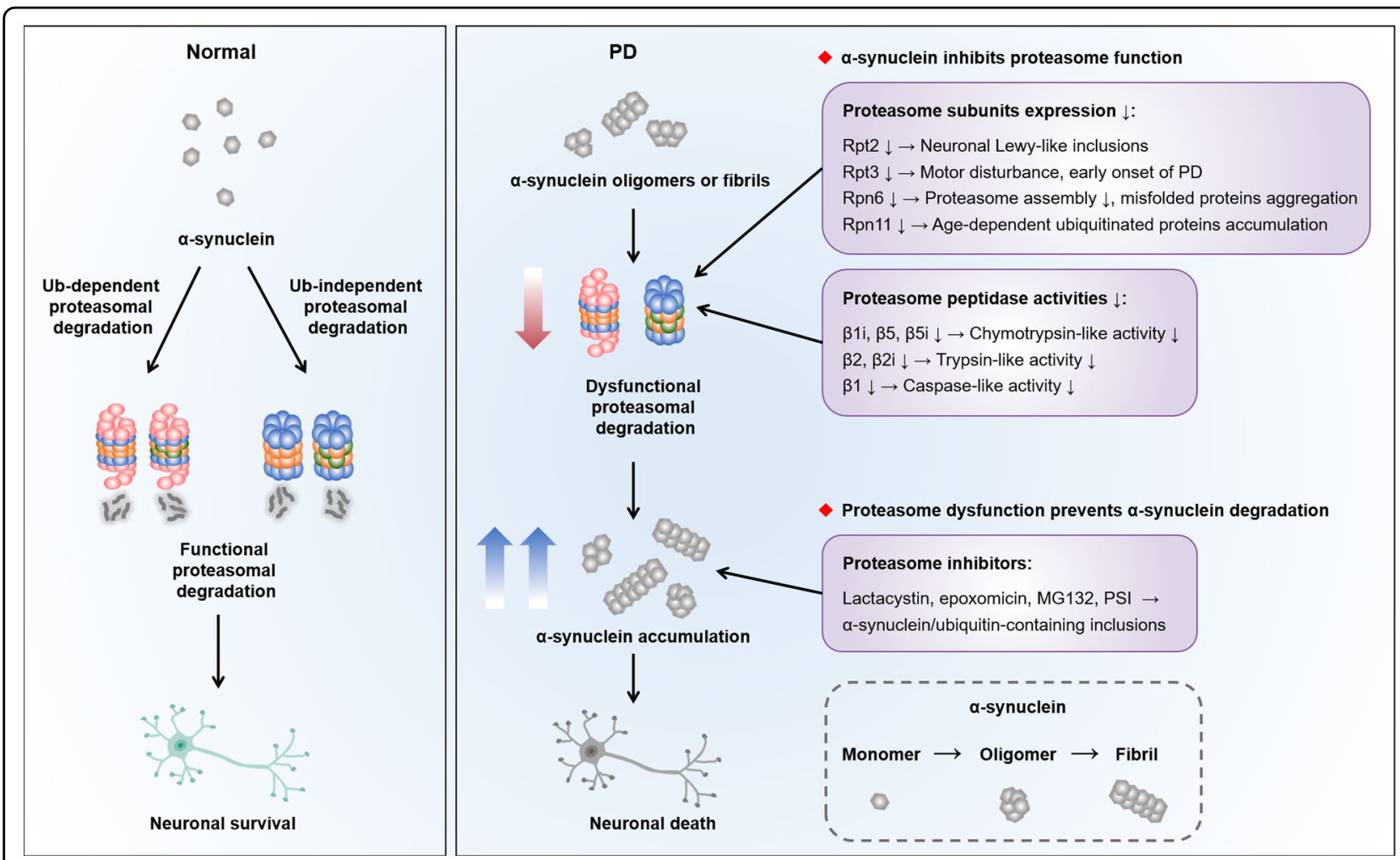

Fig. 3 The interplay between proteasome impairment and a-synuclein accumulation in PD. Under physiological state, a-synuclein is highly soluble and enriched at presynaptic terminals, which can be degraded through ubiquitin-dependent and ubiquitin-independent manner, so as to maintain the survival of neurons. In PD, a-synuclein accumulates in neuronal cell bodies to form a major component of aberrant aggregates known as Lewy bodies. a-synuclein is able to transform in different conformations, including monomers, oligomers (soluble conformations), and fibrils (insoluble conformations). The impairment of proteasome function resulting from the decreased subunits expression and proteolytic activities disturbs the degradation of substrates. Additionally, a-synuclein has been reported to inhibit the function of proteasome, which further aggravates the accumulation of a-synuclein. This suggests a vicious cycle between proteasomal impairment and proteotoxicity in PD.

endoplasmic reticulum. By contrast, autophagy coupled with lysosome is essential to degrade longer-lived macromolecules, cytosol fractions, and organelles through three autophagic pathways, macroautophagy, microautophagy, and chaperone-mediated autophagy (CMA). Indeed, maintaining proteome balance is a challenging work in the face of various external and internal stimuli owing to the age-dependent decline in the proteolysis capacity and impaired proteasomal degradation ${ }^{73}$. The resulting aberrant accumulation of misfolded and aggregated proteins probably overload the cellular ability to degrade rendering the neurons susceptible to the pathological injury (Fig. 3).

In 2001, McNaught and colleagues first reported the decreased proteasome hydrolytic activities in $\mathrm{PD}^{74}$. Specifically, in the $\mathrm{SN}$ instead of other brain regions of $\mathrm{PD}$ patients, there is a loss of $\alpha$-subunits in dopaminergic neurons and a reduction in all of three peptidase activities ${ }^{75}$. In contrast, it is probable that the reduced proteasome activity could be a consequence of the neurodegeneration, which may underly the vulnerability of the $\mathrm{SN}$ in PD.
ATPase subunit Rpt2 conditional knockout in dopaminergic neurons displays the depletion of $26 \mathrm{~S}$ proteasome, resulting in the presence of intraneuronal Lewy-like inclusions in the nigrostriatal pathway ${ }^{76}$. Besides, insertion and deletion variants in the Rpt3 gene correlate with the early onset of $\mathrm{PD}^{77}$. Conditional knockout of Rpt3 in motor neurons causes severe motor dysfunction, accompanied by progressive loss of neurons and gliosis ${ }^{78}$. Rpn11 overexpression prevents the age-dependent deficit in $26 \mathrm{~S}$ proteasome activity and ubiquitin-conjugated proteins accumulation $^{79}$. Furthermore, Rpn6 enhances the proteasome assembly and activity, which is critical for the stabilization of $\mathrm{CP}$ and the proper interaction with RP to improve age-related protein aggregates ${ }^{26,80}$. These data demonstrate that the maintenance of proteasome function is of great importance for the adequate degradation of unwanted proteins and slowing down the neurodegeneration in PD. Even with this information, it is still challenging to pinpoint the different functions produced by the multiple proteasome subunits, which is a major hurdle toward understanding the regulation of proteasome in PD. 


\section{Impairment of a-synuclein degradation through proteasome}

Emerging evidence has demonstrated that the abundant fibrillar $\alpha$-synuclein inclusions known as Lewy bodies is one of the pathological hallmarks in PD. $\alpha$-Synuclein is encoded by $S N C A$ gene, which is enriched in presynaptic terminals to modulate synaptic-vesicle trafficking, bind membranes and induce membrane curvature. Point mutations in SNCA (A30P, E46K, H50Q, G51D, A53E, and $\mathrm{A} 53 \mathrm{~T}$ ) and genomic duplications or triplications within SNCA locus lead to the autosomal dominant familial $\mathrm{PD}^{81,82}$. Indeed, $\alpha$-synuclein pathology follows a stereotypical "prion-like" propagation pattern, resulting in a cell-to-cell transmission to drive neurodegeneration in PD. Several strategies, such as using antibodies to impede the spreading of $\alpha$-synuclein and testing minute quantities of $\alpha$-synuclein in cerebrospinal fluid by seeding aggregation assay, have been implicated for PD therapies ${ }^{83,84}$. The homeostasis of $\alpha$-synuclein is maintained under intrinsic surveillance mechanisms including ubiquitin-dependent and -independent proteasomal degradation, macroautophagy and CMA. Different forms of $\alpha$-synuclein are degraded by multiple routes depending on the overall protein burden, localization and pathological states. Proteasome and macroautophagy are capable of degrading mutant $\alpha$-synuclein or intermediate oligomers, while CMA possesses a specific ability to degrade monomers and dimers of $\alpha$-synuclein ${ }^{85,86}$. Extracellular $\alpha$-synuclein is cleared by proteases, or spread among neighboring cells and eliminated within lysosomes. However, the mechanism governing whether $\alpha$-synuclein will be cleared by proteasome or autophagy remains unknown.

Treatment with proteasome inhibitors leads to the accumulation of ubiquitin and $\alpha$-synuclein immunoreactive inclusions, which supports the concept that defects in the proteasome pathway might uncover the nigral pathology in familial and sporadic forms of $\mathrm{PD}^{84-86}$. Consistent with these findings, systemic exposure to proteasome inhibitors in rats causes the formation of $\alpha$ synuclein/ubiquitin-containing inclusions resembling Lewy bodies in the remaining neurons and animals develops progressive motor deficits which closely recapitulates the main pathological features of $\mathrm{PD}^{87}$. Similar to other PD-related neurotoxins, proteasome inhibitors might offer an alternative way to model the disturbance of protein homeostasis and the chronic progressive nature of neurodegeneration as it occurs in PD. Even so, systemic administration of PSI has been challenged due to several laboratories inability to replicate the mode ${ }^{88-90}$ or reproduce only partial features such as transient motor deficits without dopaminergic neurons degeneration ${ }^{91}$ or modest depletion of striatal dopamine without motor disorder ${ }^{92}$. The impaired turnover of $\alpha$-synuclein represents a critical aspect of neurodegeneration in PD.

\section{a-Synuclein disrupts proteasome function}

Numerous studies have shown that $\alpha$-synuclein can inhibit proteasome activity, especially in the presence of oligomer or fibril species ${ }^{10}$. PC12 cells expressing A53T mutant $\alpha$-synuclein exhibit the impaired proteasomal chymotrypsin-like activity and disruption of the ubiquitin-dependent degradation system, manifested by an increase in ubiquitin-conjugated aggregates ${ }^{93}$. Their observations have been supported by another study using the same cell line expressing mutant $\alpha$-synuclein, in which the peptidase activities of proteasome are reduced resulting in mitochondrial abnormalities and neuronal death $^{94}$. Of note, proteasome function is inhibited not only by mutant $\alpha$-synuclein, but also by wild-type species. Human neuroblastoma BE-M17 cells stably expressed wild-type $\alpha$-synuclein exhibit an $\sim 50 \%$ reduction in ubiquitin-independent proteasomal degradation ${ }^{95}$. A study with yeast also demonstrates that wild-type and A30P mutant $\alpha$-synuclein impair proteasome-mediated protein degradation, but have little effects on intracellular proteasome content or protein ubiquitylation ${ }^{96}$. More recently, McKinnon $\mathrm{C}$ et al. report that $\alpha$-synuclein overexpression leads to the early-onset catalytic impairment of $26 \mathrm{~S}$ proteasome, which is associated with selective accumulation of $\alpha$-synuclein phosphorylated at Ser129 and precedes the onset of motor deficits and dopaminergic neurons degeneration ${ }^{10}$. In addition, $\alpha$ synuclein oligomers associate with the $26 \mathrm{~S}$ proteasome, leading to a significant inhibition of proteasomal activities without affecting the levels or assembly of $26 \mathrm{~S}$ proteasome $^{97}$. The proposed mechanisms of how $\alpha$-synuclein inhibits the proteasome function may be due to the direct interaction with Rpt5 or $\beta 5$ subunit $^{95,98}$. Overall, the impaired proteasome function aggravates the accumulation of $\alpha$-synuclein, which in turn binds to the proteasome, thereby inhibiting its proteolytic activity. The vicious cycle between proteasomal impairment and $\alpha$ synuclein aggregation may provide an insight into the putative neuroprotective therapies for PD.

\section{Proteasome activation-based approach for PD therapies}

Small-molecule compounds

Enhancing proteasome activity by small-molecule compounds has been regarded as a promising strategy to treat or prevent PD. USP14, a proteasome-associated DUB, contains a catalytic domain at the $\mathrm{C}$ terminus and a ubiquitin-like (Ubl) domain at the $\mathrm{N}$-terminus. The binding of USP14 to Rpn1 subunit through Ubl enhances its deubiquitinating activity, which is responsible for the removement of ubiquitin chains and prevent the release of ubiquitin to the proteolytic channel. Proteasomes lacking USP14 exhibit higher peptidase activity and ubiquitinindependent proteolysis ${ }^{99}$. In 2010, Lee et al. have 
identified IU1 as a selective small-molecule inhibitor of USP14 through high-throughput screen. IU1 treatment significantly accelerates the elimination of oxidized proteins, such as tau and TDP43, thus enhancing the resistance to oxidative stress ${ }^{100}$. Later, the improved USP14 inhibitor IU1-47, with a 10-fold more potent and retains specificity for USP14, has been found to stimulate the degradation of wild-type and pathological tau. Notably, a specific residue in tau (Lys174) is critical for IU1-47mediated tau degradation ${ }^{101}$ (Table 3). Presumably, further studies are needed to determine the effects of USP14 inhibitors on the proteasomal degradation of $\alpha$-synuclein in PD.

Critical proteins that unfold and aggregate in neurodegenerative diseases, such as $\alpha$-synuclein, tau, huntingtin $(\mathrm{Htt})$, and polyglutamine androgen receptor (polyQ AR), are client proteins of heat shock protein 90 (HSP90). HSP90/HSP70-based chaperone machinery has been identified as a key regulator of proteostasis owing to its role in the protein quality control ${ }^{102}$. Nonetheless, HSP90 and HSP70 possess opposing effects on client proteins stability. HSP90 prevents the degradation of proteins through the ubiquitin-proteasome pathway, whereas HSP70 promotes ubiquitylation for proteasomal degradation dependent on E3 ubiquitin ligase $\mathrm{C}$ terminus of Hsc70-interacting protein (CHIP). This indicates that an effective way to eliminate the aggregation of neurotoxic proteins may be to inhibit HSP90 or promote HSP70 function ${ }^{103}$. Among the compounds that inhibit HSP90, geldanamycin acts as a nucleotide to inhibit the intrinsic ATPase activity of the chaperone ${ }^{104}$. It has been reported that geldanamycin facilitates the degradation of oligomeric $\alpha$-synuclein, phosphorylated tau, and Htt aggregates in vivo ${ }^{105-107}$. The less toxic analogue of geldanamycin, 17-AAG, has improved brain permeability, which inhibits the formation of $\alpha$-synuclein oligomer and rescues cytotoxicity mediated by secreted $\alpha$-synuclein ${ }^{108}$. Furthermore, 17-AAG can reduce tau pathology and attenuate $A \beta$-induced synaptic toxicity and memory impairment ${ }^{109-111}$. YM-1 allosterically stimulates the activity of HSP70 and enhances its binding to unfolded substrates. Treatment of YM-1 promotes the clearance of polyglutamine proteins and increase CHIP-dependent ubiquitylation of neuronal nitric oxide synthase ${ }^{112,113}$ (Table 3). The compounds that promote HSP70dependent proteasomal degradation in combination with HSP90 inhibitors might be beneficial for the clearance of aggregated proteins, which should be tested in PD models.

\section{Transcriptional activation of proteasome expression}

Considering that the formation of proteasome is energetically costly, the fine tuning mechanisms are required to maintain sufficient amount of proteasomes to combat the proteotoxicity. Transcription factor NRF2 has been proved to reduce steady-state levels of $\alpha$-synuclein, shorten its half-life in part by accelerating the degradation of $\alpha$-synuclein ${ }^{114}$. Pharmacological activation of NRF2 by dimethyl fumarate (DMF), a drug already in use for multiple sclerosis (MS), significantly reduces $\alpha$-synuclein aggregates and rescues neurons from oxidative stressinduced injury ${ }^{115,116}$. Several naturally occurring NRF2 activators, such as sulforaphane, epigallocatechin-3gallate (EGCG), and curcumin, stimulate NRF2 signaling pathway to regulate proteostasis. Sulforaphane activates protein degradation machineries and promotes mutant Htt degradation via proteasome ${ }^{117}$. EGCG can inhibit $\alpha$ synuclein fibrillation and aggregation, as well as protect PC12 cells against $\alpha$-synuclein-mediated toxicity ${ }^{118}$. Additionally, curcumin has been found to prevent the aggregation of $\alpha$-synuclein in the dopaminergic neurons ${ }^{119,120}$. A novel curcumin analog, called ASC-JM17, is characterized to strikingly activate NRF1 and NRF2, which further increases the expression of proteasome subunits so as to mitigate the proteotoxicity in spinal and bulbar muscular atrophy (SBMA) and other polyglutamine diseases ${ }^{121}$. Moreover, lcariin functions as an inducer of NRF1 by increasing the expression of HRD1, an ER-anchoring E3 ubiquitin ligase, and protect neurons from ER stress-induced apoptosis in PC12 cells ${ }^{122}$. Recently, high-throughput chemical screen has identified pharmacological agonists of NRF1, that is, sCGA884 and sCIN027. Co-treatment of these activators and MG-132 induce a dramatic transcriptional activity of NRF1. However, there exists an important limitation that single treatment of sCGA884 or sCIN027 leads to only partially efficacious NRF1 transcriptional activation ${ }^{123}$. In this case, a more refined understanding about the upstream signaling governing the transcriptional activity as well as the downstream effects on proteasome subunits expression is critical for targeting proteasome in the treatment of PD.

\section{Modulation of proteasome phosphorylation}

The phosphorylation status of proteasome subunits by PKA, p38 MAPK, and c-Abl has been discussed to modulate the degradation of substrates via proteasome. Administration of rolipram, a specific phosphodiesterase type 4 (PDE4) inhibitor and PKA activator, enhances $26 \mathrm{~S}$ proteasome activity, leading to the lower levels of aggregated tau and improved cognitive performance ${ }^{124}$ (Table 3). Later, a proteasome activity-based probe detects 11 novel compounds that enhance proteasome activity, with the p38 MAPK inhibitor PD169316 being one of the most potent molecules. Furthermore, chemical and genetic inhibition of p38 MAPK, its upstream kinases ASK1 and MKK6, and its downstream target MK2, all remarkably increase proteasome activity and the degradation of ubiquitylated proteins as well as $\alpha$-synuclein ${ }^{125}$. These findings may provide a strategy for investigating the 


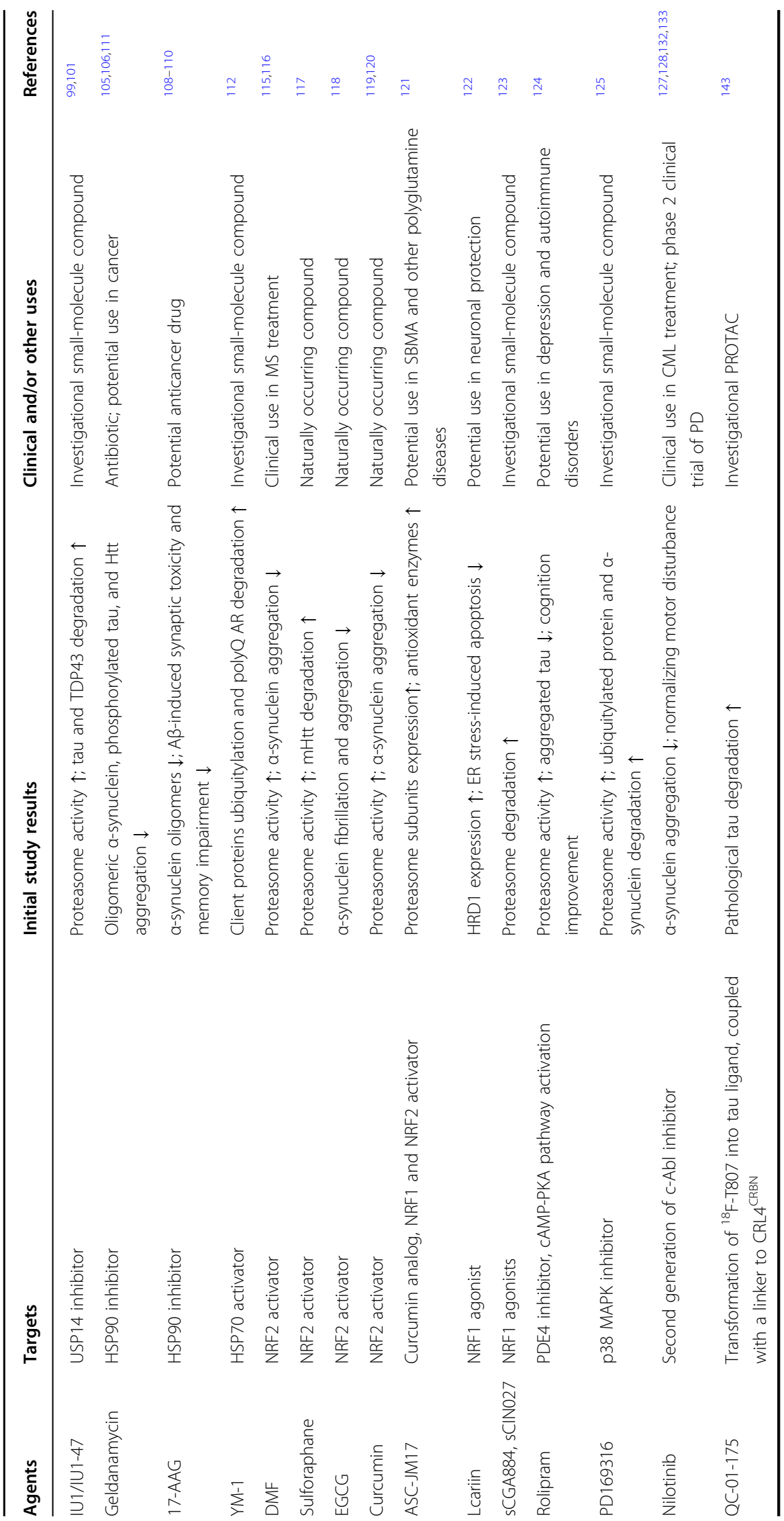


complex biology of p38 MAPK to reduce the aberrant protein aggregates under proteotoxic stress.

The activity of c-Abl, a nonreceptor tyrosine kinase, is increased in the SN of PD postmortem brains and animal models, followed by the accumulation of pathological $\alpha$ synuclein ${ }^{126,127}$. Several studies have identified $\alpha$-synuclein and parkin as the substrates of c-Abl, which specifically phosphorylates $\alpha$-synuclein at Tyr39 and parkin at Tyr143. Currently, the second-generation c-Abl inhibitors Nilotinib and Bafetinib, clinically used in the treatment of chronic myeloid leukemia (CML), have been reported to reduce the accumulation of $\alpha$-synuclein and reverse the degeneration of nigral dopaminergic neurons in PD animal models ${ }^{128-131}$. Recently, in a phase 2 randomized clinical trial with 75 patients, 150 and $300 \mathrm{mg}$ doses of Nilotinib administered orally once daily for 12 months appear to be reasonably safe, and the dopamine metabolites in cerebrospinal fluid are significantly increased $^{132,133}$. Intriguingly, $150 \mathrm{mg}$ but not $300 \mathrm{mg}$ of Nilotinib has shown a reduction in $\alpha$-synuclein oligomers $^{132}$ (Table 3). As a consequence, a definitive phase 3 study will be conducted to evaluate the effects of Nilotinib or exploring other c-Abl inhibitors as a potential disease-modifying drug in PD.

\section{Augmentation of proteasome assembly}

The transmembrane domain recognition complex (TRC) pathway, which mediates the insertion of tail-anchored proteins, has been implicated to regulate $\mathrm{CP}$ assembly. Bag6, a protein in TRC pathway, facilitates the incorporation of $\beta$-subunits into $\alpha$-ring or directly associates with precursor RP subunits, which is involved in the effective degradation of proteins ${ }^{134}$. In addition, genome-wide functional screening has characterized inactive rhomboid protein 1 (iRhom1), a member of the rhomboid-like family of proteases, as a novel stimulator of proteasome activity $^{135,136}$. Under ER stress, iRhom1 interacts with $20 \mathrm{~S} \mathrm{CP}$ assembly chaperones PAC1 and PAC2, affecting their protein stability and dimerization. iRhom 1 is induced by ER stressors, such as thapsigargin and tunicamycin, and iRhom1 overexpression has been found to significantly enhance the function of proteasome and mitigate the rough-eye phenotype of mutant Huntingtin ${ }^{136}$. However, the precise mechanisms by which TRC pathway controls the proteasome $\mathrm{CP}$ assembly or iRhom1 regulates the stability of PAC1/PAC2 dimers remain to be established. NRF3, a close homologue of NRF1, directly augments the expression of proteasome maturation protein (POMP), consequently enhancing the degradation of p53 in a ubiquitin-independent manner ${ }^{137}$. In addition, $19 \mathrm{~S}$ base subunit Rpn6 also regulates RP-CP association through the direct binding to $\alpha 2$ subunit. Ectopic expression of Rpn6 is sufficient to enhance proteasome activity and improve resistance to proteotoxic stress ${ }^{138}$.

\section{PROTAC}

PROTAC operates via an event-driven mechanism, which couples a small-molecule binder of a target protein to an E3 ubiquitin ligase via a flexible chemical linker, thereby eliciting ectopic ubiquitylation and eventually leading to the proteasomal degradation ${ }^{139}$. PROTAC is initially implicated in the recruitment of the androgen receptor to the E3 ubiquitin ligase MDM2, and androgen receptor degradation is proteasome dependent, which is mitigated in cells pretreated with proteasome inhibitor ${ }^{140}$. More recently, the field of targeting proteins degradation has expanded dramatically, and PROTACs have been found to be more selective than the intrinsic inhibitors $^{141,142}$. Although p38 MAPK inhibition is implicated to reduce the aberrant protein aggregates under proteotoxic stress, none have displayed the safety and tolerability capable of receiving FDA approval. A recent work has developed p38 MAPK-selective PROTAC based on a single small-molecule binder (foretinib) and E3 ubiquitin ligase von Hippel-Lindau (VHL), which selectively degrades $\mathrm{p} 38 \alpha$ MAPK via proteasome ${ }^{15}$.

Another work designs and synthesizes an effective tau degrader, namely QC-01-175, in which tau positron emission tomography (PET) tracer ${ }^{18} \mathrm{~F}-\mathrm{T} 807$ is transformed into pathogenic tau ligand, coupled with a linker to the CRL4 ${ }^{\text {CRBN }}$ E3 ubiquitin ligase complex. Intriguingly, QC-01-175 preferentially degrades pathological tau, indicating the degrader specificity for disease-relevant forms of $\operatorname{tau}^{143}$ (Table 3). PROTAC may be utilized to develop a functional $\alpha$-synuclein degrader for the targeted degradation of pathological $\alpha$-synuclein species in PD where high-quality PET tracers are available. Despite the cytosolic and nuclear proteins can routinely be degraded, the degradation of protein in both the Golgi and ER via PROTAC has not been reported ${ }^{144}$. Indeed, if a PROTAC is designed to induce the degradation of a protein within one system, then it can be applied more widely in different cell types without genetic modification. Regarding clinical applications, off-target effects and appropriate dosage may be an issue, as saturating doses of PROTAC can antagonize the binding of PROTAC-protein complexes to their ternary partner, a well-described phenomenon known as the hook effect in cell assays ${ }^{145}$. The likelihood of successful PROTAC development represents a promising strategy to advance our understanding of neurodegenerative disease and translate those insights into targeted therapies.

\section{Conclusions}

The recent advances in the field of proteasome remarkably improve our understanding of its biological function, well-organized assembly and dynamic regulation of proteasome homeostasis. Under specific conditions, the dissociation and reassembly of subunits to form different 
types of proteasome, and the distinct function of singly or doubly capped proteasomes need to be further elucidated. In PD, there exists a large number of mutant or misfolded proteins aggregates, which is a testament to the importance of proteasome for proteostasis and its potential as a therapeutic target. Although a variety of proteasome activation strategies have been identified, no drugs that directly enhance proteasome function are available. The elucidation of other compounds to intensify proteasomal degradation will open up new possibilities for PD treatment based on proteasome modulation.

\section{Acknowledgements}

We thank Prof. Lingqiang Zhang at National Center for Protein Sciences (Beijing) for his valuable guidance and support for this manuscript.

\section{Author contributions}

M.B. concepted and wrote the manuscript; M.B. and Q.J. created and edited the figures; X.D. and X.C. outlined the review and supervised the text; H.J. conceived and edited the final work. All authors have read and approved the final version of the manuscript.

\section{Conflict of interest}

The authors declare that they have no conflict of interest.

\section{Ethics statement}

This paper has been approved by the Ethics Committee of Qingdao University.

\section{Funding}

This work was supported by the National Natural Science Foundation of China (31771110, 31701020, 81701063, 81801264), Shandong Province Natural Science Foundation (ZR2019ZD31, ZR2020QH125), National Key Research and Development Program of China (2016YFC1306501), China Postdoctoral Science Foundation (2020M671991), Key Research and Development Program of Shandong Province (2018GSF118042), Taishan Scholars Construction Project, Innovative Research Team of High-Level Local Universities in Shanghai, Shandong Province Postdoctoral Innovative Project, and Postdoctoral Applied Research Project in Qingdao.

\section{Publisher's note}

Springer Nature remains neutral with regard to jurisdictional claims in published maps and institutional affiliations.

Received: 16 September 2020 Revised: 13 January 2021 Accepted: 15 January 2021

Published online: 04 February 2021

\section{References}

1. Hough, R., Pratt, G. \& Rechsteiner, M. Purification of two high molecular weight proteases from rabbit reticulocyte lysate. J. Biol. Chem. 262, 8303-8313 (1987).

2. Waxman, L., Fagan, J. M. \& Goldberg, A. L. Demonstration of two distinct high molecular weight proteases in rabbit reticulocytes, one of which degrades ubiquitin conjugates. J. Biol. Chem. 262, 2451-2457 (1987).

3. Collins, G. A. \& Goldberg, A. L. The logic of the 265 proteasome. Cell 169, 792-806 (2017).

4. Bard, J. A. M., Bashore, C., Dong, K. C. \& Martin, A. The 265 proteasome utilizes a kinetic gateway to prioritize substrate degradation. Cell 177, 286-298. e215 (2019).

5. Yu, H. \& Matouschek, A. Recognition of client proteins by the proteasome. Annu. Rev. Biophys. 46, 149-173 (2017).

6. Dahlmann, B. Mammalian proteasome subtypes: their diversity in structure and function. Arch. Biochem. Biophys. 591, 132-140 (2016).
7. Xie, S. C. et al. The structure of the PA28-20S proteasome complex from Plasmodium falciparum and implications for proteostasis. Nat. Microbiol. 4, 1990-2000 (2019).

8. Guo, X., Huang, X. \& Chen, M. J. Reversible phosphorylation of the $26 \mathrm{~S}$ proteasome. Protein Cell 8, 255-272 (2017).

9. Dantuma, N. P. \& Bott, L. C. The ubiquitin-proteasome system in neurodegenerative diseases: precipitating factor, yet part of the solution. Front. Mol. Neurosci. 7, 70 (2014).

10. McKinnon, $\mathrm{C}$. et al. Early-onset impairment of the ubiquitin-proteasome system in dopaminergic neurons caused by alpha-synuclein. Acta Neuropathol. Commun. 8, 17 (2020).

11. Thibaudeau, T. A. \& Smith, D. M. A practical review of proteasome pharmacology. Pharmacol. Rev. 71, 170-197 (2019).

12. Mishra, R., Upadhyay, A., Prajapati, V. K. \& Mishra, A. Proteasome-mediated proteostasis: novel medicinal and pharmacological strategies for diseases. Med. Res. Rev. 38, 1916-1973 (2018).

13. Burslem, G. M. \& Crews, C. M. Proteolysis-targeting chimeras as therapeutics and tools for biological discovery. Cell 181, 102-114 (2020).

14. Paiva, S. L. \& Crews, C. M. Targeted protein degradation: elements of PROTAC design. Curr. Opin. Chem. Biol. 50, 111-119 (2019).

15. Smith, B. E. et al. Differential PROTAC substrate specificity dictated by orientation of recruited E3 ligase. Nat. Commun. 10, 131 (2019).

16. Yao, L. et al. The proteasome activator REGgamma counteracts immunoproteasome expression and autoimmunity. J. Autoimmun. 103, 102282 (2019).

17. Li, S. et al. Regulation of c-Myc protein stability by proteasome activator REGgamma. Cell Death Differ. 22, 1000-1011 (2015).

18. Hayashi, M., Ishibashi, T., Tanaka, K. \& Kasahara, M. The mouse genes encoding the third pair of beta-type proteasome subunits regulated reciprocally by IFN-gamma: structural comparison, chromosomal localization, and analysis of the promoter. J. Immunol. 159, 2760-2770 (1997).

19. Murata, S. et al. Regulation of CD8+ T cell development by thymus-specific proteasomes. Science 316, 1349-1353 (2007).

20. Uechi, H., Hamazaki, J. \& Murata, S. Characterization of the testis-specific proteasome subunit alpha4s in mammals. J. Biol. Chem. 289, 12365-12374 (2014).

21. Mannhaupt, G., Schnall, R., Karpov, V., Vetter, I. \& Feldmann, H. Rpn4p acts as a transcription factor by binding to PACE, a nonamer box found upstream of 265 proteasomal and other genes in yeast. FEBS Lett. 450, 27-34 (1999).

22. Xie, Y. \& Varshavsky, A. RPN4 is a ligand, substrate, and transcriptional regulator of the 265 proteasome: a negative feedback circuit. Proc. Natl Acad. Sci. USA 98, 3056-3061 (2001).

23. Shirozu, R., Yashiroda, H. \& Murata, S. Identification of minimum Rpn4responsive elements in genes related to proteasome functions. FEBS Lett. 589, 933-940 (2015).

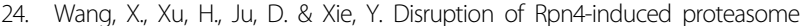
expression in Saccharomyces cerevisiae reduces cell viability under stressed conditions. Genetics 180, 1945-1953 (2008).

25. $\mathrm{Xu}, \mathrm{H}$. et al. The CCAAT box-binding transcription factor NF-Y regulates basal expression of human proteasome genes. Biochim. Biophys. Acta $\mathbf{1 8 2 3}$ 818-825 (2012).

26. Vilchez, D. et al. Increased proteasome activity in human embryonic stem cells is regulated by PSMD11. Nature 489, 304-308 (2012).

27. Vangala, J. R., Dudem, S., Jain, N. \& Kalivendi, S. V. Regulation of PSMB5 protein and beta subunits of mammalian proteasome by constitutively activated signal transducer and activator of transcription 3 (STAT3): potential role in bortezomib-mediated anticancer therapy. J. Biol. Chem. 289 12612-12622 (2014).

28. Kwak, M. K., Wakabayashi, N., Greenlaw, J. L., Yamamoto, M. \& Kensler, T. W. Antioxidants enhance mammalian proteasome expression through the Keap1-Nrf2 signaling pathway. Mol. Cell Biol. 23, 8786-8794 (2003).

29. Pickering, A. M., Linder, R. A., Zhang, H., Forman, H. J. \& Davies, K. J. Nrf2dependent induction of proteasome and Pa28alphabeta regulator are required for adaptation to oxidative stress. J. Biol. Chem. 287, 10021-10031 (2012).

30. Radhakrishnan, S. K et al. Transcription factor Nrf1 mediates the proteasome recovery pathway after proteasome inhibition in mammalian cells. Mol. Cell 38, 17-28 (2010).

31. Lee, C. S. et al. Loss of nuclear factor E2-related factor 1 in the brain leads to dysregulation of proteasome gene expression and neurodegeneration. Proc Natl Acad. Sci. USA 108, 8408-8413 (2011). 
32. James, A. B., Conway, A. M. \& Morris, B. J. Regulation of the neuronal proteasome by Zif268 (Egr1). J. Neurosci. 26, 1624-1634 (2006).

33. Zanelli, E., Zhou, P., Cao, H., Smart, M. K. \& David, C. S. Genomic organization and tissue expression of the mouse proteasome gene Lmp-7. Immunogenetics 38, 400-407 (1993).

34. Cruz, M., Elenich, L. A., Smolarek, T. A., Menon, A. G. \& Monaco, J. J. DNA sequence, chromosomal localization, and tissue expression of the mouse proteasome subunit Imp10 (Psmb10) gene. Genomics 45, 618-622 (1997).

35. Rousseau, A. \& Bertolotti, A. An evolutionarily conserved pathway controls proteasome homeostasis. Nature 536, 184-189 (2016).

36. Chantranupong, L. \& Sabatini, D. M. Cell biology: The TORC1 pathway to protein destruction. Nature 536, 155-156 (2016).

37. Zhang, Y. et al. Coordinated regulation of protein synthesis and degradation by mTORC1. Nature 513, 440-443 (2014).

38. Haass, C. \& Kloetzel, P. M. The Drosophila proteasome undergoes changes in its subunit pattern during development. Exp. Cell Res. 180, 243-252 (1989).

39. Pereira, M. E. \& Wilk, S. Phosphorylation of the multicatalytic proteinase complex from bovine pituitaries by a copurifying CAMP-dependent protein kinase. Arch. Biochem. Biophys. 283, 68-74 (1990).

40. Zhang, F. et al. Proteasome function is regulated by cyclic AMP-dependent protein kinase through phosphorylation of Rpt6. J. Biol. Chem. 282, 22460-22471 (2007).

41. Lokireddy, S., Kukushkin, N. V. \& Goldberg, A. L. CAMP-induced phosphorylation of 265 proteasomes on Rpn6/PSMD11 enhances their activity and the degradation of misfolded proteins. Proc. Natl Acad. Sci. USA 112, E7176-E7185 (2015).

42. VerPlank, J. J. S., Lokireddy, S., Zhao, J. \& Goldberg, A. L. 265 Proteasomes are rapidly activated by diverse hormones and physiological states that raise CAMP and cause Rpn6 phosphorylation. Proc. Natl Acad. Sci. USA 116 4228-4237 (2019).

43. Myeku, N., Wang, H. \& Figueiredo-Pereira, M. E. cAMP stimulates the ubiquitin/proteasome pathway in rat spinal cord neurons. Neurosci. Lett. 527, 126-131 (2012).

44. Djakovic, S. N., Schwarz, L. A., Barylko, B., DeMartino, G. N. \& Patrick, G. N Regulation of the proteasome by neuronal activity and calcium/calmodulindependent protein kinase II. J. Biol. Chem. 284, 26655-26665 (2009).

45. Bingol, B. et al. Autophosphorylated CaMKllalpha acts as a scaffold to recruit proteasomes to dendritic spines. Cell 140, 567-578 (2010).

46. Hamilton, A. M. et al. Activity-dependent growth of new dendritic spines is regulated by the proteasome. Neuron 74, 1023-1030 (2012).

47. Djakovic, S. N. et al. Phosphorylation of Rpt6 regulates synaptic strength in hippocampal neurons. J. Neurosci. 32, 5126-5131 (2012).

48. Jarome, T. J., Kwapis, J. L., Ruenzel, W. L. \& Helmstetter, F. J. CaMKIl, but not protein kinase A, regulates Rpt6 phosphorylation and proteasome activity during the formation of long-term memories. Front. Behav. Neurosci. 7, 115 (2013).

49. Jarome, T. J., Ferrara, N. C., Kwapis, J. L. \& Helmstetter, F. J. CaMKII regulates proteasome phosphorylation and activity and promotes memory destabilization following retrieval. Neurobiol. Learn Mem. 128, 103-109 (2016).

50. Guo, X. et al. Site-specific proteasome phosphorylation controls cell proliferation and tumorigenesis. Nat. Cell Biol. 18, 202-212 (2016).

51. Banerjee, S. et al. Ancient drug curcumin impedes $26 \mathrm{~S}$ proteasome activity by direct inhibition of dual-specificity tyrosine-regulated kinase 2. Proc. Nat Acad. Sci. USA 115, 8155-8160 (2018).

52. Lee, S. H., Park, Y., Yoon, S. K. \& Yoon, J. B. Osmotic stress inhibits proteasome by p38 MAPK-dependent phosphorylation. J. Biol. Chem. 285, 41280-41289 (2010).

53. Liu, X. et al. Interaction between c-Abl and Arg tyrosine kinases and proteasome subunit PSMA7 regulates proteasome degradation. Mol. Cell 22, 317-327 (2006).

54. Li, D. et al. c-Abl regulates proteasome abundance by controlling the ubiquitin-proteasomal degradation of PSMA7 subunit. Cell Rep. 10, 484-496 (2015).

55. Wani, P. S., Suppahia, A., Capalla, X., Ondracek, A. \& Roelofs, J. Phosphorylation of the C-terminal tail of proteasome subunit alpha7 is required for binding of the proteasome quality control factor Ecm29. Sci. Rep. 6, 27873 (2016).

56. Crosas, B. et al. Ubiquitin chains are remodeled at the proteasome by opposing ubiquitin ligase and deubiquitinating activities. Cell 127, 1401-1413 (2006).

57. Isasa, M. et al. Monoubiquitination of RPN10 regulates substrate recruitment to the proteasome. Mol. Cell 38, 733-745 (2010).
58. Besche, H. C. et al. Autoubiquitination of the $26 \mathrm{~S}$ proteasome on Rpn13 regulates breakdown of ubiquitin conjugates. EMBO J. 33, 1159-1176 (2014).

59. Schmitt, S. M. et al. Involvement of ALAD-20S proteasome complexes in ubiquitination and acetylation of proteasomal alpha2 subunits. J. Cell Biochem. 117, 144-151 (2016).

60. Ullrich, O. et al. Poly-ADP ribose polymerase activates nuclear proteasome to degrade oxidatively damaged histones. Proc. Natl Acad. Sci. USA 96, 6223-6228 (1999).

61. Catalgol, B. et al. Chromatin repair after oxidative stress: role of PARPmediated proteasome activation. Free Radic. Biol. Med. 48, 673-680 (2010).

62. Ullrich, O. et al. Turnover of oxidatively damaged nuclear proteins in BV-2 microglial cells is linked to their activation state by poly-ADP-ribose polymerase. FASEB J. 15, 1460-1462 (2001).

63. Wells, L., Vosseller, K. \& Hart, G. W. Glycosylation of nucleocytoplasmic proteins: signal transduction and O-GlcNAc. Science 291, 2376-2378 (2001).

64. Liu, K. et al. Accumulation of protein O-GICNAc modification inhibits proteasomes in the brain and coincides with neuronal apoptosis in brain areas with high O-GlcNAc metabolism. J. Neurochem. 89, 1044-1055 (2004).

65. Zhang, F. et al. O-GlcNAc modification is an endogenous inhibitor of the proteasome. Cell 115, 715-725 (2003)

66. Overath, T. et al. Mapping of O-GlcNAc sites of $20 \mathrm{~S}$ proteasome subunits and Hsp90 by a novel biotin-cystamine tag. Mol. Cell Proteomics 11, 467-477 (2012)

67. Wang, D. et al. Regulation of acetylation restores proteolytic function of diseased myocardium in mouse and human. Mol. Cell Proteom. 12 3793-3802 (2013).

68. Swatek, K. N. \& Komander, D. Ubiquitin modifications. Cell Res. 26, 399-422 (2016).

69. Ohtake, F. et al. Ubiquitin acetylation inhibits polyubiquitin chain elongation EMBO Rep. 16, 192-201 (2015).

70. Lehtonen, S., Sonninen, T. M., Wojciechowski, S., Goldsteins, G. \& Koistinaho, J. Dysfunction of cellular proteostasis in Parkinson's disease. Front. Neurosci. 13 457 (2019).

71. Hipp, M. S., Kasturi, P. \& Hartl, F. U. The proteostasis network and its decline in ageing. Nat. Rev. Mol. Cell Biol. 20, 421-435 (2019).

72. Klaips, C. L., Jayaraj, G. G. \& Hartl, F. U. Pathways of cellular proteostasis in aging and disease. J. Cell Biol. 217, 51-63 (2018).

73. Kurtishi, A., Rosen, B., Patil, K. S., Alves, G. W. \& Moller, S. G. Cellular proteostasis in neurodegeneration. Mol. Neurobiol. 56, 3676-3689 (2019).

74. McNaught, K. S. \& Jenner, P. Proteasomal function is impaired in substantia nigra in Parkinson's disease. Neurosci. Lett. 297, 191-194 (2001).

75. McNaught, K. S., Belizaire, R., Isacson, O., Jenner, P. \& Olanow, C. W. Altered proteasomal function in sporadic Parkinson's disease. Exp. Neurol. 179, 38-46 (2003).

76. Bedford, L. et al. Depletion of 265 proteasomes in mouse brain neurons causes neurodegeneration and Lewy-like inclusions resembling human pale bodies. J. Neurosci. 28, 8189-8198 (2008).

77. Wahl, C. et al. A comprehensive genetic study of the proteasomal subunit S6 ATPase in German Parkinson's disease patients. J. Neural Transm. 115 1141-1148 (2008).

78. Tashiro, Y. et al. Motor neuron-specific disruption of proteasomes, but not autophagy, replicates amyotrophic lateral sclerosis. J. Biol. Chem. 287 42984-42994 (2012)

79. Tonoki, A. et al. Genetic evidence linking age-dependent attenuation of the 265 proteasome with the aging process. Mol. Cell Biol. 29, 1095-1106 (2009).

80. Pathare, G. R. et al. The proteasomal subunit Rpn6 is a molecular clamp holding the core and regulatory subcomplexes together. Proc. Natl Acad. Sci. USA 109, 149-154 (2012).

81. Polymeropoulos, M. H. et al. Mutation in the alpha-synuclein gene identified in families with Parkinson's disease. Science 276, 2045-2047 (1997).

82. Bi, M., Kang, S., Du, X., Jiao, Q. \& Jiang, H. Association between SNCA rs356220 polymorphism and Parkinson's disease: a meta-analysis. Neurosci. Lett. 717, 134703 (2020)

83. Tran, H. T. et al. Alpha-synuclein immunotherapy blocks uptake and templated propagation of misfolded alpha-synuclein and neurodegeneration Cell Rep. 7, 2054-2065 (2014)

84. Kang, U. J. et al. Comparative study of cerebrospinal fluid alpha-synuclein seeding aggregation assays for diagnosis of Parkinson's disease. Mov. Disord. 34, 536-544 (2019)

85. Stefanis, L. et al. How is alpha-synuclein cleared from the cell? J. Neurochem. 150, 577-590 (2019) 
86. Wong, Y. C. \& Krainc, D. alpha-synuclein toxicity in neurodegeneration: mechanism and therapeutic strategies. Nat. Med. 23, 1-13 (2017).

87. McNaught, K. S., Perl, D. P., Brownell, A. L. \& Olanow, C. W. Systemic exposure to proteasome inhibitors causes a progressive model of Parkinson's disease. Ann. Neurol. 56, 149-162 (2004).

88. Kadoguchi, N., Kimoto, H., Yano, R., Kato, H. \& Araki, T. Failure of acute administration with proteasome inhibitor to provide a model of Parkinson's disease in mice. Metab. Brain Dis. 23, 147-154 (2008).

89. Kordower, J. H. et al. Failure of proteasome inhibitor administration to provide a model of Parkinson's disease in rats and monkeys. Ann. Neurol. 60 264-268 (2006).

90. Mathur, B. N., Neely, M. D., Dyllick-Brenzinger, M., Tandon, A. \& Deutch, A. Y. Systemic administration of a proteasome inhibitor does not cause nigrostriatal dopamine degeneration. Brain Res. 1168, 83-89 (2007).

91. Landau, A. M., Kouassi, E., Siegrist-Johnstone, R. \& Desbarats, J. Proteasome inhibitor model of Parkinson's disease in mice is confounded by neurotoxicity of the ethanol vehicle. Mov. Disord. 22, 403-407 (2007).

92. Hirst, S. J. \& Ferger, B. Systemic proteasomal inhibitor exposure enhances dopamine turnover and decreases dopamine levels but does not affect MPTP-induced striatal dopamine depletion in mice. Synapse $\mathbf{6 2}, \mathbf{8 5 - 9 0}$ (2008).

93. Stefanis, L., Larsen, K. E., Rideout, H. J., Sulzer, D. \& Greene, L. A Expression of A53T mutant but not wild-type alpha-synuclein in PC12 cells induces alterations of the ubiquitin-dependent degradation system, loss of dopamine release, and autophagic cell death. J. Neurosci. 21, 9549-9560 (2001)

94. Tanaka, Y. et al. Inducible expression of mutant alpha-synuclein decreases proteasome activity and increases sensitivity to mitochondria-dependent apoptosis. Hum. Mol. Genet. 10, 919-926 (2001).

95. Snyder, $\mathrm{H}$. et al. Aggregated and monomeric alpha-synuclein bind to the $\mathbf{S 6}$ proteasomal protein and inhibit proteasomal function. J. Biol. Chem. 278, 11753-11759 (2003).

96. Chen, Q., Thorpe, J. \& Keller, J. N. Alpha-synuclein alters proteasome function, protein synthesis, and stationary phase viability. J. Biol. Chem. 280 30009-30017 (2005).

97. Emmanouilidou, E., Stefanis, L. \& Vekrellis, K. Cell-produced alpha-synuclein oligomers are targeted to, and impair, the 265 proteasome. Neurobiol. Aging 31, 953-968 (2010)

98. Lindersson, E. et al. Proteasomal inhibition by alpha-synuclein filaments and oligomers. J. Biol. Chem. 279, 12924-12934 (2004).

99. Kim, H. T. \& Goldberg, A. L. The deubiquitinating enzyme Usp14 allosterically inhibits multiple proteasomal activities and ubiquitin-independent proteolysis. J. Biol. Chem. 292, 9830-9839 (2017).

100. Lee, B. H. et al. Enhancement of proteasome activity by a small-molecule inhibitor of USP14. Nature 467, 179-184 (2010).

101. Boselli, M. et al. An inhibitor of the proteasomal deubiquitinating enzyme USP14 induces tau elimination in cultured neurons. J. Biol. Chem. 292, 19209-19225 (2017).

102. Imai, J., Maruya, M., Yashiroda, H., Yahara, I. \& Tanaka, K. The molecular chaperone $\mathrm{Hsp} 90$ plays a role in the assembly and maintenance of the $26 \mathrm{~S}$ proteasome. EMBO J. 22, 3557-3567 (2003).

103. Boland, B. et al. Promoting the clearance of neurotoxic proteins in neurodegenerative disorders of ageing. Nat. Rev. Drug Discov. 17, 660-688 (2018).

104. Roe, S. M. et al. Structural basis for inhibition of the Hsp90 molecular chaperone by the antitumor antibiotics radicicol and geldanamycin. J. Med. Chem. 42, 260-266 (1999).

105. McLean, P. J., Klucken, J., Shin, Y. \& Hyman, B. T. Geldanamycin induces Hsp70 and prevents alpha-synuclein aggregation and toxicity in vitro. Biochem. Biophys. Res. Commun. 321, 665-669 (2004).

106. Sittler, A. et al. Geldanamycin activates a heat shock response and inhibits huntingtin aggregation in a cell culture model of Huntington's disease. Hum. Mol. Genet. 10, 1307-1315 (2001).

107. Auluck, P. K., Meulener, M. C. \& Bonini, N. M. Mechanisms of suppression of \{alpha\}-synuclein neurotoxicity by geldanamycin in Drosophila. J. Biol. Chem. 280, 2873-2878 (2005).

108. Danzer, K. M. et al. Heat-shock protein 70 modulates toxic extracellular alphasynuclein oligomers and rescues trans-synaptic toxicity. FASEB J. 25, 326-336 (2011).

109. Chen, Y. et al. Hsp90 chaperone inhibitor 17-AAG attenuates Abetainduced synaptic toxicity and memory impairment. J. Neurosci. 34 2464-2470 (2014).
110. Ho, S. W. et al. Effects of 17-allylamino-17-demethoxygeldanamycin (17-AAG) in transgenic mouse models of frontotemporal lobar degeneration and Alzheimer's disease. Transl. Neurodegener. 2, 24 (2013).

111. Putcha, P. et al. Brain-permeable small-molecule inhibitors of Hsp90 prevent alpha-synuclein oligomer formation and rescue alpha-synuclein-induced toxicity. J. Pharmacol. Exp. Ther. 332, 849-857 (2010).

112. Wang, A. M. et al. Activation of Hsp70 reduces neurotoxicity by promoting polyglutamine protein degradation. Nat. Chem. Biol. 9, 112-118 (2013).

113. Roodveldt, C. et al. Chaperone proteostasis in Parkinson's disease: stabilization of the Hsp70/alpha-synuclein complex by Hip. EMBO J. 28, 3758-3770 (2009).

114. Skibinski, G. et al. Nrf2 mitigates LRRK2- and alpha-synuclein-induced neurodegeneration by modulating proteostasis. Proc. Natl Acad. Sci. USA 114 1165-1170 (2017).

115. Campolo, M. et al. The neuroprotective effect of dimethyl fumarate in an MPTP-mouse model of Parkinson's disease: involvement of reactive oxygen species/nuclear factor-kappaB/nuclear transcription factor related to NF-E2. Antioxid. Redox Signal 27, 453-471 (2017).

116. Lastres-Becker, I. et al. Repurposing the NRF2 activator dimethyl fumarate as therapy against synucleinopathy in Parkinson's disease. Antioxid. Redox Signal 25, 61-77 (2016).

117. Liu, Y. et al. Sulforaphane enhances proteasomal and autophagic activities in mice and is a potential therapeutic reagent for Huntington's disease. J. Neurochem. 129, 539-547 (2014).

118. Teng, Y., Zhao, J., Ding, L., Ding, Y. \& Zhou, P. Complex of EGCG with Cu(II) suppresses amyloid aggregation and Cu(ll)-induced cytotoxicity of alphasynuclein. Molecules 24, 2940 (2019).

119. Sharma, N. \& Nehru, B. Curcumin affords neuroprotection and inhibits alphasynuclein aggregation in lipopolysaccharide-induced Parkinson's disease model. Inflammopharmacology 26, 349-360 (2018).

120. Ahmad, B. \& Lapidus, L. J. Curcumin prevents aggregation in alpha-synuclein by increasing reconfiguration rate. J. Biol. Chem. 287, 9193-9199 (2012).

121. Bott, L. C. et al. A small-molecule Nrf1 and Nrf2 activator mitigates polyglutamine toxicity in spinal and bulbar muscular atrophy. Hum. Mol. Genet. 25, 1979-1989 (2016).

122. Li, F., Gao, B., Dong, H., Shi, J. \& Fang, D. Icariin induces synoviolin expression through NFE2L1 to protect neurons from ER stress-induced apoptosis. PLoS ONE 10, e0119955 (2015).

123. laconelli, J. et al. Small-molecule stimulators of NRF1 transcriptional activity. Chembiochem 21, 1816-1819 (2019).

124. Myeku, N. et al. Tau-driven $26 \mathrm{~S}$ proteasome impairment and cognitive dysfunction can be prevented early in disease by activating CAMP-PKA signaling. Nat. Med. 22, 46-53 (2016).

125. Leestemaker, Y. et al. Proteasome activation by small molecules. Cell Chem Biol. 24, 725-736 e727 (2017)

126. Brahmachari, S. et al. Activation of tyrosine kinase c-Abl contributes to alpha-synuclein-induced neurodegeneration. J. Clin. Invest. 126, 2970-2988 (2016).

127. Mahul-Mellier, A. L. et al. c-Abl phosphorylates alpha-synuclein and regulates its degradation: implication for alpha-synuclein clearance and contribution to the pathogenesis of Parkinson's disease. Hum. Mol. Genet. 23, 2858-2879 (2014).

128. Hebron, M. L., Lonskaya, I. \& Moussa, C. E. Nilotinib reverses loss of dopamine neurons and improves motor behavior via autophagic degradation of alphasynuclein in Parkinson's disease models. Hum. Mol. Genet. 22, 3315-3328 (2013).

129. Karuppagounder, S. S. et al. The c-Abl inhibitor, nilotinib, protects dopaminergic neurons in a preclinical animal model of Parkinson's disease. Sci. Rep. 4, 4874 (2014)

130. Tanabe, A. et al. A novel tyrosine kinase inhibitor AMN107 (nilotinib) normalizes striatal motor behaviors in a mouse model of Parkinson's disease. Front. Cell Neurosci. 8, 50 (2014).

131. Imam, S. Z. et al. Neuroprotective efficacy of a new brain-penetrating C-Abl inhibitor in a murine Parkinson's disease model. PLOS ONE 8, e65129 (2013).

132. Pagan, F. L. et al. Nilotinib effects on safety, tolerability, and potential biomarkers in Parkinson disease: a phase 2 randomized clinical trial. JAMA Neurol. 77, 309-317 (2019)

133. Pagan, F. et al. Nilotinib effects in Parkinson's disease and dementia with Lewy bodies. J. Parkinsons Dis. 6, 503-517 (2016).

134. Akahane, T., Sahara, K., Yashiroda, H., Tanaka, K. \& Murata, S. Involvement of Bag6 and the TRC pathway in proteasome assembly. Nat. Commun. 4, 2234 (2013). 
135. Freeman, M. The rhomboid-like superfamily: molecular mechanisms and biological roles. Annu. Rev. Cell Dev. Biol. 30, 235-254 (2014).

136. Lee, W. et al. iRhom1 regulates proteasome activity via PAC1/2 under ER stress. Sci. Rep. 5, 11559 (2015).

137. Kobayashi, A. \& Waku, T. New addiction to the NRF2-related factor NRF3 in cancer cells: ubiquitin-independent proteolysis through the 205 proteasome. Cancer Sci. 111, 6-14 (2020).

138. Vilchez, D. et al. RPN-6 determines C. elegans longevity under proteotoxic stress conditions. Nature 489, 263-268 (2012).

139. Raina, K. \& Crews, C. M. Targeted protein knockdown using small molecule degraders. Curr. Opin. Chem. Biol. 39, 46-53 (2017).

140. Schneekloth, A. R., Pucheault, M., Tae, H. S. \& Crews, C. M. Targeted intracellular protein degradation induced by a small molecule: En route to chemical proteomics. Bioorg. Med. Chem. Lett. 18, 5904-5908 (2008).

141. Bondeson, D. P. et al. Lessons in PROTAC design from selective degradation with a promiscuous warhead. Cell Chem. Biol. 25, 78-87 e75 (2018).

142. Schapira, M., Calabrese, M. F., Bullock, A. N. \& Crews, C. M. Targeted protein degradation: expanding the toolbox. Nat. Rev. Drug Discov. 18, 949-963 (2019).

143. Silva, M. C. et al. Targeted degradation of aberrant tau in frontotemporal dementia patient-derived neuronal cell models. elife 8, e45457 (2019).
144. Smith, M. H., Ploegh, H. L. \& Weissman, J. S. Road to ruin: targeting proteins for degradation in the endoplasmic reticulum. Science 334, 1086-1090 (2011).

145. Olson, C. M. et al. Pharmacological perturbation of CDK9 using selective CDK9 inhibition or degradation. Nat. Chem. Biol. 14, 163-170 (2018).

146. Liu, X. et al. Reversible phosphorylation of Rpn1 regulates 265 proteasome assembly and function. Proc. Natl Acad. Sci. USA 117, 328-336 (2020).

147. Bose, S., Stratford, F. L., Broadfoot, K. I., Mason, G. G. \& Rivett, A. J. Phosphorylation of $20 S$ proteasome alpha subunit C8 (alpha7) stabilizes the 265 proteasome and plays a role in the regulation of proteasome complexes by gamma-interferon. Biochem. J. 378, 177-184 (2004).

148. Zmijewski, J. W., Banerjee, S. \& Abraham, E. S-glutathionylation of the Rpn2 regulatory subunit inhibits $26 \mathrm{~S}$ proteasomal function. J. Biol. Chem. 284, 22213-22221 (2009).

149. Silva, G. M. et al. Redox control of 205 proteasome gating. Antioxid. Redox Signal 16, 1183-1194 (2012).

150. Leme, J. M. M. et al. Mutations of Cys and Ser residues in the alpha5subunit of the 205 proteasome from Saccharomyces cerevisiae affects gating and chronological lifespan. Arch. Biochem. Biophys. 666, 63-72 (2019). 